\title{
Functional characterization of SAG/RBX2/ROC2/RNF7, an antioxidant protein and an E3 ubiquitin ligase
}

\author{
Yi Sun ${ }^{\bowtie}$, Hua Li \\ Division of Radiation and Cancer Biology, Department of Radiation Oncology, University of Michigan, 4424B Medical Science-I, \\ 1301 Catherine Street, Ann Arbor, MI 48109, USA. \\ $\triangle$ Correspondence: sunyi@umich.edu \\ Received October 6, 2012 Accepted October 9, 2012
}

\begin{abstract}
SAG (Sensitive to Apoptosis Gene), also known as RBX2 (RING box protein 2), ROC2 (Regulator of Cullins 2), or RNF7 (RING Finger Protein 7), was originally cloned in our laboratory as a redox inducible antioxidant protein and later characterized as the second member of the RBX/ROC RING component of the SCF (SKP1-CUL-F-box Proteins) E3 ubiquitin ligase. When acting alone, SAG scavenges oxygen radicals by forming inter- and intra- molecular disulfide bonds, whereas by forming a complex with other components of the SCF E3 ligase, SAG promotes ubiquitination and degradation of a number of protein substrates, includ-

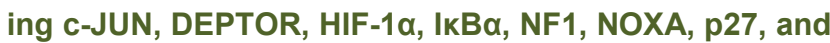
procaspase-3, thus regulating various signaling pathways and biological processes. Specifically, SAG protects cells from apoptosis, confers radioresistance, and plays an essential and non-redundant role in mouse embryogenesis and vasculogenesis. Furthermore, stress-inducible SAG is overexpressed in a number of human cancers and SAG overexpression correlates with poor patient prognosis. Finally, SAG transgenic expression in epidermis causes an early stage inhibition, but later stage promotion, of skin tumorigenesis triggered by DMBA/TPA. Given its major role in promoting targeted degradation of tumor suppressive proteins, leading to apoptosis suppression and accelerated tumorigenesis, SAG E3 ligase appears to be an attractive anticancer target.
\end{abstract}

KEYWORDS antioxidant, angiogenesis, apoptosis, Cullin-RING ligases, radiation resistance, reactive oxygen species, SAG/RBX2/ROC2/RNF7, SCF E3 ligases, tumorigenesis, ubiquitin ligase, vasculogenesis

\section{INTRODUCTION}

SAG (Sensitive to Apoptosis Gene), also known as RBX2 (RING box protein-2), ROC2 (Regulator of cullins-2), or RNF7 (RING finger protein-7) belongs to an evolutionarily conserved gene family with $96 \%$ sequence identity between human and mouse and $55 \%$ between human and yeast (Sun et al., 2001; Sun, 2008). SAG is expressed ubiquitously in human tissues with a very high expression in heart, skeletal muscle, and testis, three organs with high levels of oxygen consumption. At the subcellular level, SAG is expressed in both cytoplasm and nucleus (Duan et al., 1999). Human SAG is mapped onto chromosome $3 q 22-24$ with three splicing variants and two family pseudogenes (Swaroop et al., 2001), whereas mouse Sag was mapped onto chromosome 9 with three exons and two introns (Tan et al., 2011b). Structurally, both human SAG and mouse Sag encode a protein of 113 amino acids, of which 12 are cysteine residues (Duan et al., 1999) (Fig. 1). At the carboxyl portion of SAG protein, there is a $\mathrm{C}_{3} \mathrm{H}_{2} \mathrm{C}_{3}$ motif, which chelates two zinc atoms to form the RING domain (Fig. 2), a characteristic of a domain with E3 ubiquitin ligase activity (Lipkowitz and Weissman, 2011). This primary structure of SAG determines that SAG is a dual-functional molecule. Biochemically, when acting alone, SAG has non-enzymatic antioxidant activity which scavenges reactive oxygen species at the expense of self-oligomerization via formation of intra- and inter-molecule disulfide bonds (Swaroop et al., 1999). When forming the complex with other components of SCF (Skp1-Cullin1-F-box proteins), SAG, like its family member, RBX1/ROC1, has intrinsic E3 


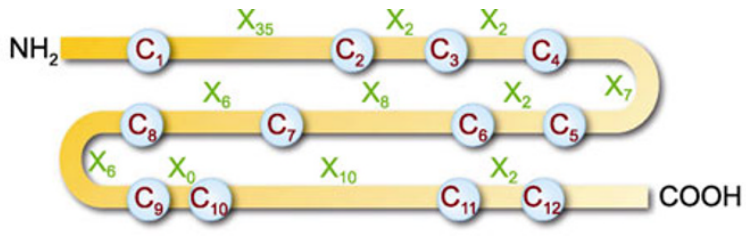

Figure 1. A schematic presentation of cysteine residues on SAG. A total of 12 cysteine residues are shown with $X$ standing for any other amino acids. The numeric number stands for the number of amino acids between two cysteine residues. It is unclear, however, which cysteine residues would form intra-molecular or inter-molecular disulfide bonds. Note that the figure was not drawn to scale.

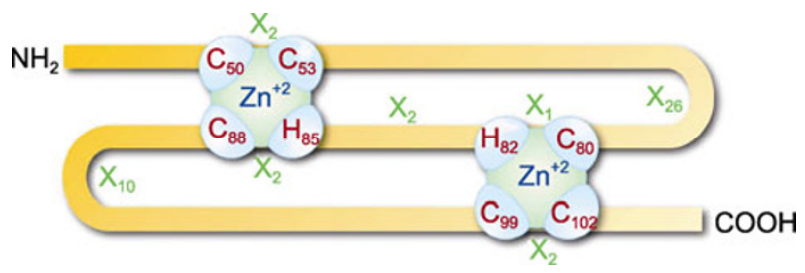

Figure 2. A schematic presentation of the RING finger domain on SAG. Shown is the $\mathrm{C}_{3} \mathrm{H}_{2} \mathrm{C}_{3}$ RING finger domain that binds to two zinc atoms in a cross-braced arrangement with the codon number of each cysteine or histidine residue indicated. The $X$ stands for any amino acid, whereas the numeric number stands for the number of amino acids between two cysteine or histidine residues. Note that the figure was not drawn to scale.

ligase activity (Swaroop et al., 2000; Tan et al., 2010). Below we will review extensively SAG's biochemical activities and biological functions, as characterized by the use of biochemical assays, in vitro cell culture and in vivo animal models.

\section{BIOCHEMICAL PROPERTIES}

\section{SAG as an antioxidant protein}

Antioxidant defense systems that scavenge reactive oxygen species (ROS) consist mainly of 1) antioxidant enzymes, including superoxide dismutase, catalase, glutathione peroxidase, and glutathione reductase; 2) antioxidant proteins (e.g. thioredoxin, metallothionein); and 3) small molecule antioxidants (e.g. GSH, N-acetyl-L-cysteine, dithiothreitol, vitamin C, and vitamin E) (Sun, 1990). Like other thiol-based antioxidant proteins, such as thioredoxin and metallothionein (Sato and Bremner, 1993; Deneke, 2000; Viarengo et al., 2000), SAG has more than $10 \%$ cysteine residues in its composition and has the capacity to generate thiol/disulfide redox buffer and chelate metals, such as zinc and copper (Duan et al., 1999; Swaroop et al., 1999; Sun et al., 2001).

Our early study showed that bacterially expressed and purified SAG protein is a redox sensitive protein, which forms oligomers upon storage at $4^{\circ} \mathrm{C}$. SAG oligomerization was rapidly induced by hydrogen peroxide in dose and incubation time dependent manners, which can be blocked by the small molecule antioxidant dithiothreitol (DTT), or by $N$-ethylmaleimide (NEM), an alkylating agent, indicating that SAG oligomerization is caused by the formation of inter- or intra-molecular disulfide bonds, although involvement of each individual cysteine residue in the formation of these disulfide bonds cannot be precisely defined (Swaroop et al., 1999). Furthermore, SAG also binds to metal ions, including zinc, iron and copper, and inhibits copper-induced lipid peroxidation in test tube (Duan et al., 1999; Swaroop et al., 1999). SAG also prevents lipid peroxidation induced by 2,2-azo-bis2-amidinopropane hydrochloride, a free radical generator, without metal ion involvement (Frei et al., 1988). SAG-induced inhibition of lipid peroxidation is completely abolished by the pretreatment of SAG with the alkylating agent NEM, suggesting that the free sulfhydryl groups of cysteines on the SAG molecule are the major contributors to this activity (Duan et al., 1999). Given the observations that metallothionein, a cysteine-rich, but RING finger-less protein also has such an activity (Duan et al., 1999) and that some SAG RING finger mutants still retain this activity (Swaroop et al., 1999; Sun et al., 2001), it is possible that the RING finger domain may not be directly involved in ROS scavenging activity of SAG. Thus, the cysteine residues (which comprises $10.6 \%$ of SAG protein), rather than the RING finger motif (with six cysteine residues involved) (see Figs. 1 and 2 for comparison) per se are mainly responsible for observed ROS scavenging activity, although it is hard to differentiate them. Taken together, our results demonstrate that SAG is an antioxidant and metal chelating protein that scavenges ROS at the expense of self-oligomerization.

Other investigators have subsequently shown that SAG possesses a potent peroxidase activity to decompose hydrogen peroxide in the presence of dithiothreitol, which can again be completely abrogated by NEM, suggesting that the sulfhydryl groups of cysteines on the SAG molecule could function as strong nucleophiles to destroy hydrogen peroxide. Furthermore, the peroxidase activity of SAG can be extended to t-butyl-hydroperoxide and fatty acid hydroperoxide (Kim, 2002). The same group of investigators further found that SAG significantly inhibits single strand breaks induced by synthesized peroxynitrite and 3-morphol-inosydnomine $\mathrm{N}$-ethylcarbamide (SIN-1) in supercoiled plasmid DNA. Formation of 8-hydroxy-2-deoxyguanosine in calf thymus DNA by peroxynitrite and SIN-1 is also significantly inhibited by SAG. Again, the protective effect of SAG on peroxynitrite-mediated DNA damage was completely abolished by NEM (Kim, 2003a).

Most recently, two studies show an antioxidant protective role of SAG in cultured cells and even in vivo mouse brains. When directly added to the culture medium, purified cell-penetrable SAG protein (Tat-SAG) protects astrocytes from oxidative stress-induced death (Kim et al., 2010). More 
interestingly, when administered intraperitoneally into mice, Tat-SAG significantly reduces in a dose dependent manner DNA damage and lipid peroxidation in mouse neurons (Kim et al., 2010; Yoo et al., 2012), and prevents neuronal cell death triggered by transient forebrain ischemia (Kim et al., 2010). Finally, we showed recently that ES cells with Sag deletion have an increased steady-state level of ROS upon radiation exposure which contributes to an enhanced sensitivity to radiation (Tan et al., 2010). Taken together, these in vitro and in vivo studies clearly demonstrate that antioxidant SAG inhibits ROS-induced lipid peroxidation and DNA damage, and consequently protects cells from ROS-induced death.

\section{SAG as a RING component of SCF E3 ubiquitin ligase}

Normal function of eukaryotic cells depends upon a fine balance between synthesis and degradation of proteins. Many short-lived and biologically important proteins need to be rapidly degraded upon completion of their designated functions. The protein degradation is mainly executed by the ubiquitin-dependent proteasome system in which a targeted protein is ubiquitinated by three step-wise enzymatic reactions involving E1 ubiquitin activating enzyme, E2 ubiquitin conjugating enzyme, and E3 ubiquitin ligase, which recognizes the target substrate and catalyzes the transfer of ubiquitin from an E2 to an internal lysine residue of a substrate. Multiple runs of the E1-E2-E3 mediated reaction lead to poly-ubiquitination of the targeted protein, which is then recognized, if poly-ubiquitin chains are linked via lysine 48 (K48), by the $26 \mathrm{~S}$ proteasome for targeted degradation (Ciechanover, 1998; Hershko and Ciechanover, 1998; Jia and Sun, 2011) (Fig. 3).

The human genome encodes two E1 enzymes, about 38 E2 enzymes, and greater than 600 distinct E3 enzymes with each of them having the potential to recognize multiple substrates, leading to targeted ubiquitination and degradation of thousands of protein substrates (Li et al., 2008). Four major types of E3 ubiquitin ligases have been defined: the $\mathrm{N}$-end rule E3s, HECT (Homology to E6AP C-Terminus) containing E3s, RING (Really Interesting New Gene) containing E3s, and APC/C (Anaphase promoting complex/cyclosome) E3s (Hershko and Ciechanover, 1998; Hershko et al., 2000). Finally, the E3 ubiquitin ligases can be a single peptide (such as MDM2 or XIAP) or consist of multiple components (such as SCF or $\mathrm{APC} / \mathrm{C})$.

Among all E3s, CRLs (Cullin-RING ligases) with the SCF (SKP1-Cullin 1-F-box proteins) as the founding members, are the largest E3 ligases in number. They promote timely ubiqutination and degradation of many key proteins, thus playing important roles in many processes including cell cycle progression, oncogenesis, signal transduction, gene transcription, embryonic development, genomic integrity and tumor suppression (Nakayama and Nakayama, 2006; Deshaies and Joazeiro, 2009; Jia and Sun, 2011). The components of

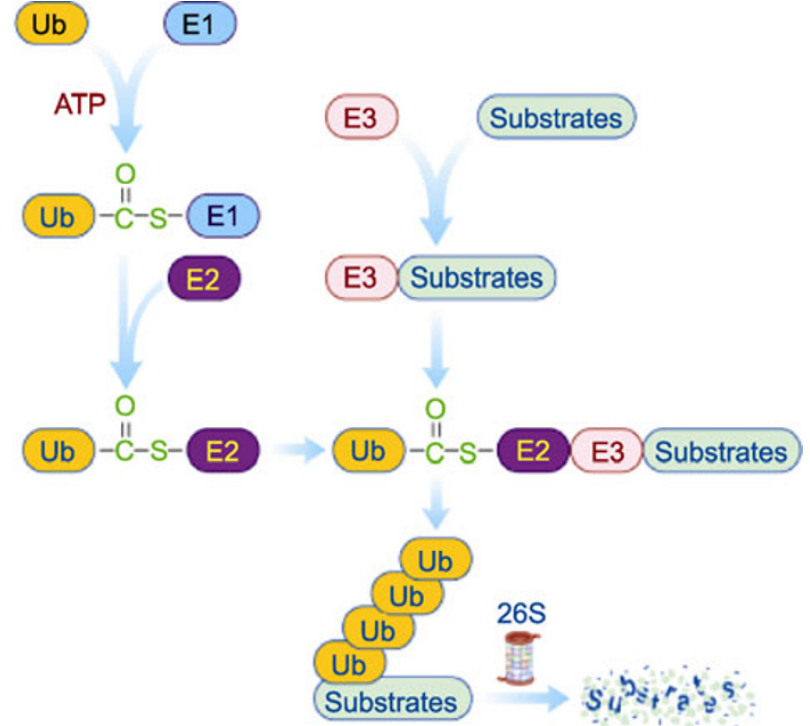

Figure 3. Ubiquitination reaction. In the presence of ATP, ubiquitin is activated by E1 ubiquitin activating enzyme through a thioester-bond between the C-terminus glycine (G76) of ubiquitin and a cysteine residue at the active site of E1. Activated ubiquitin is then transferred to E2 ubiquitin conjugating enzyme, again through the thioester bond between the G76 of ubiquitin and a cysteine residue at the active site of E2. Finally, ubiquitin is covalently attached to a target protein through an isopeptide bond between $\mathrm{G} 76$ of ubiquitin and the $\varepsilon$ amino group of an internal lysine residue of the target protein, catalyzed by an E3 ubiquitin ligase E3, which recognizes the specific substrate. Multiple runs of this reaction lead to polyubiquitination of a substrate, resulting in recognition by the $26 \mathrm{~S}$ proteasome for targeted degradation.

the CRLs include few members of adaptor proteins such as SKP1 and elongin B/C; two members of the RING family proteins, RBX1/ROC1 and SAG/RBX2/ROC2 (Wei and Sun, 2010), eight members of scaffold family protein cullins (Cul -1, $-2,-3,-4 A,-4 B,-5,-7$, and -9) (Sarikas et al., 2011) and many family members of substrate receptor proteins (Lee and Zhou, 2010; Sarikas et al., 2011). The SCF E3, also known as CRL1, consists of SKP1, RBX1 or SAG/RBX2, cullin-1, and one of 69 members of the F-box family proteins (Jin et al., 2004). The F-box proteins determine substrate specificity, whereas cullin-1-RBX1/SAG forms the core E3 ligase that transfers the ubiquitin from the E2 to the substrate (Wu et al., $2000 \mathrm{~b})$. Another unique feature for substrate ubiquitination and degradation mediated by CRL1/SCF E3s is that a substrate has to be phosphorylated by a kinase(s) prior to being recognized by an F-box protein (Willems et al., 2004; Deshaies and Joazeiro, 2009).

To determine potential E3 ubiquitin ligase activity of SAG, we purified SAG-Cul-1 complex by immunoprecipitation after transient transfection of plasmids expressing SAG and Cul-1. In a ligase reaction mixture containing ubiquitin, E1, E2, ATP, and SAG-Cul-1 complex, we observed the formation of high 
molecular weight smears, indicative of polyubiquitination in the E1 and E2 dependent manner (Swaroop et al., 2000). SAG-Cul-1 mediated polyubiquitination is also dependent on the intact SAG RING structure, since the replacement of wild type SAG with SAG RING mutants completely abrogate this reaction (Swaroop et al., 2000). The ligase activity of SAG-Cul-1 is similar to the RBX1-Cul-1 complex which was included as a positive control (Swaroop et al., 2000). Furthermore, we observed that SAG-Cul-1 and RBX-Cul-1 have a similar ligase activity in promoting polyubiquitination of phosphorylated IKB in an in vitro ubiquitination assay (Tan et al., 2010). Thus, like its family member RBX1, SAG has intrinsic E3 ubiquitin ligase activity, when it has formed a complex with cullin-1. It is worth noting that SAG binds to the $\beta$-subunit of caseine kinase II (CK2) and is phosphorylated at the $\mathrm{Thr}^{10}$ residue by CK2 (Son et al., 1999; Kim, 2003b). SAG phosphorylation may change its ligase activity, as evidenced by accumulation of $1 \mathrm{~KB} \alpha$ and p27 when a non-phosphorylatable SAG-T10A (threonine 10 is replaced by alanine) was overexpressed (Kim, 2003b). However, using both SAG-T10A and phosphor-mimicking mutant SAG-T10E (threonine 10 is replaced by glutamate), we found that while SAG-wt and SAG-T10A mutant have a similar protein half-life of $10 \mathrm{~h}$, the half-life of SAG-T10E mutant is much shortened to $\sim 2 \mathrm{~h}$, suggesting that SAG phosphorylation at the $\mathrm{Thr}^{10}$ may significantly reduce its stability. All three forms of SAG have similar ligase activity, suggesting that SAG phosphorylation has no effect on its intrinsic E3 ligase activity (He et al., 2007).

Consistent with SAG as an E3 ubiquitin ligase, the following cellular proteins have been identified and characterized as the substrates of SAG-SCF E3 ubiquitin ligase.

\section{Procaspase-3}

SAG was first identified as an anti-apoptotic protein (Duan et al., 1999; Sun, 1999). After demonstration that SAG has E3 ubiquitin ligase activity in addition to being an antioxidant protein, we attempted to understand the potential ligase-dependent mechanism(s) by which SAG protects cells from apoptosis. We found that SAG binds to pro-caspase-3, a prozymogen form of caspase- 3 which, as an effector caspase upon activation, plays a key role in committing cells to apoptosis (Cryns and Yuan, 1998; Earnshaw et al., 1999). The SAG-procaspase-3 binding is mediated through an F-box protein, $\beta$-TrCP, which recognizes the first 38 amino acids of pro-caspase-3. The in vitro ubiquitination assay showed that SAG- $\beta$-TrCP promotes ubiquitination of pro-caspase-3. Ectopic expression of $\beta$-TrCP shortens protein half-life of procaspase-3, whereas siRNA silencing of $\beta$-TrCP or SAG causes its accumulation; the later results in an increased caspase- 3 activity upon activation by etoposide and TRAIL and sensitization of human cancer cells to these anti-cancer agents (Tan et al., 2006). Thus, SAG- $\beta$-TrCP mediated pro-caspase-3 degradation likely contributes to SAG's anti-apoptotic function.
C-Jun

SAG was initially identified as a redox-inducible gene (Sun, 1997; Duan et al., 1999). Our subsequent study revealed that SAG is also inducible by TPA through the mechanism involving transcription factor AP-1, a heterodimer of c-Jun and Fos that regulates many biological process through the transactivation of its downstream targets (Angel and Karin, 1991; Shaulian and Karin, 2002). We found several consensus AP-1 binding motifs in the promoter of the SAG gene. Upon TPA exposure, c-Jun and c-Fos are induced to form AP-1, which binds to these sites and transactivates SAG expression ( $\mathrm{Gu}$ et al., 2007b). Since c-Jun is a previously known ubiquitination target of SCF ${ }^{\mathrm{FBXW} 7}$ (Nateri et al., 2004; Wei et al., 2005), we determined potential involvement of SAG and found that SAG siRNA silencing significantly inhibits c-Jun ubiquitination and blocks c-Jun degradation induced by FBXW7, leading to c-Jun accumulation. Likewise, ectopic SAG expression blocks c-Jun accumulation induced by TPA, which is abrogated completely by a proteasome inhibitor, MG132 (Gu et al., 2007b). These results strongly suggest that c-Jun is a substrate of SAG-SCF ${ }^{\mathrm{FBXW7}} \mathrm{E3}$, and that SAG-c-Jun establishes an auto-feedback loop. Upon induction by c-Jun/AP-1, SAG recruits FBXW7 to promote c-Jun degradation, thus shutting down the AP-1 signal to keep it in check.

\section{HIF-1 $\alpha$}

In a search for additional agents or stress conditions that would induce SAG expression, we found that hypoxia is a potent SAG inducer. Hypoxia-induced SAG expression occurs at the transcription level and is mediated by transcription factor HIF-1, a heterodimer of HIF-1 $\alpha$ and $\mathrm{HIF}-1 \beta$, which promotes angiogenesis, proliferation and cell survival by transactivating many of its target genes involved in regulation of these processes (Semenza, 2003). We identified a perfect $\mathrm{HIF}-1$ binding consensus sequence in the intron 1 of the SAG gene, which is required for HIF-1-mediated SAG transactivation (Tan et al., 2008). Since HIF-1a is a well-known substrate of CRL2 ${ }^{\mathrm{VHL}}$ E3 ligase (Maxwell et al., 1999; Ivan et al., 2001; Jaakkola et al., 2001), we determined potential involvement of SAG and found that ectopically expressed SAG promotes VHL-mediated HIF-1a ubiquitination and degradation. Likewise, SAG siRNA silencing inhibits VHL-induced HIF-1 $\alpha$ degradation and promotes HIF-1 $\alpha$ accumulation upon hypoxia. Further study revealed that although VHL mainly binds to Cul2/RBX1, it is also associated with SAG/Cul5, particularly under hypoxic conditions, which facilitates HIF-1 $\alpha$ degradation (Tan et al., 2008). Thus, analogous to the SAG-C-JUN axis, SAG-HIF-1a also establishes an auto-feedback loop in which SAG, upon induction by hypoxia/HIF-1, promotes HIF-1a degradation to shut down the HIF-1 signals in timely fashion. 


\section{IKBa}

IKBa is a cytoplasmic protein with a well-known function as the inhibitor of NFKB, a p50/p65 heterodimer transcription factor (Baeuerle and Baltimore, 1988a). Through binding to NFkB, IкB retains p50/p65 in the cytoplasm, thus preventing nuclear translocation of NFKB to act as a pleiotropic transcription factor (Baeuerle and Baltimore, 1988b). It is well-established that IKBa is a substrate of $\mathrm{SCF}^{\beta \operatorname{TrCP}}$ (Fuchs et al., 1999; Winston et al., 1999). The involvement of SAG in the process is, however, previously unknown. We found that during the late stage of skin carcinogenesis induced by DMBA/TPA, SAG-transgenic expression in epidermis causes IKBa degradation and subsequent p65 nuclear translocation and NFKB activation. As a result, DMBA/TPA-induced tumors derived from SAG-transgenic expression are bigger in size with remarkably reduced apoptosis (Gu et al., 2007a). In a separate cell culture model, we also found that IkBa protein accumulates in Sag-null mouse embryonic stem cells, which prevents NFKB activation triggered by ionizing radiation or TNFa. Consistently, ectopic SAG expression shortens IkBa protein half-life upon TNFa stimulation and directly promotes the ubiquitination of phosphorylated IKBa (Tan et al., 2010). Thus, IKBa is a direct substrate of SAG-SCF ${ }^{\beta \operatorname{TrCP}}$ E3 (Tan et al., 2010), in addition to RBX1-SCF ${ }^{\beta T r C P} E 3$ (Wu et al., 2000a). $A$ recent protein microarray study in attempt to identify the interactome of NEMO, a $48-\mathrm{kDa}$ protein that acts as an essential modulator of NFKB (Yamaoka et al., 1998), identify SAG as a NEMO binding protein (Fenner et al., 2010). Although it is unclear whether or how SAG-NEMO interaction affects NFKB activity, this study did show that SAG overexpression stimulates, whereas SAG siRNA silencing reduces, NFKB transcriptional activation (Fenner et al., 2010). Thus, SAG can modulate NFKB activity at multiple levels.

\section{NF1}

NF1 (Neurofibromatosis-1) is a tumor suppressor gene which is frequently mutated in many types of sporadic human cancers (Cichowski and Jacks, 2001; Le and Parada, 2007). Patients with NF1 mutations are predisposed to developing a variety of benign and malignant tumors mainly in the peripheral and central nervous system (Cichowski and Jacks, 2001). Nf1 contains a functional domain of RAS GTPase Activating Protein (GAP) and through which Nf1 inhibits RAS, a powerful oncogene that promotes cell proliferation, angiogenesis and tumorigenesis (Kranenburg et al., 2004; Dhillon et al., 2007), by accelerating conversion of active RAS-GTP to inactive RAS-GDP (Le and Parada, 2007). In our mechanistic study of embryonic death induced by Sag knockout, we found that Nf1 is accumulated in Sag-null embryonic stem cells during endothelial differentiation, which prevents the formation of cystic embryoid bodies (Tan et al., 2011b). Subsequent rescue experiments showed that this differentiation defect can be completely abrogated upon simultaneous dele- tion of $\mathrm{Nf} 1$, indicating that $\mathrm{Nf1}$ accumulation plays a causal role (Tan et al., 2011b). We followed up this lead and found that SAG binds to Nf1 through an $F$ box protein FBXW7 (Welcker and Clurman, 2008) via an evolutionarily conserved FBXW7 binding motif on Nf1. Mutations on this motif abrogate FBXW7 binding and subsequent Nf1 ubiquitination. Furthermore, ectopic expression of SAG and FBXW7 promotes Nf1 ubiquitination and degradation, and shortens Nf1 protein half-life, whereas SAG or FBXW7 deletion extended Nf1 protein half-life. Thus, Nf1 is a bona fide substrate of SAG-SCF ${ }^{\text {FBXW7 }}$ (Tan et al., 2011b). Figure 4 captures in cartoon form how SAG-SCF ${ }^{\text {FBXW7 }}$ promotes Nf1 degradation leading to RAS activation.

\section{Others}

Although less well characterized, we have identified other potential substrates of SAG-SCF E3, as follows.

a) p27, a well-characterized inhibitor of cyclin dependent kinase that blocks cell cycle progression at the $\mathrm{G} 1$ phase (Sherr and Roberts, 1995, 1999). p27 has been previously characterized as a substrate of SCF ${ }^{\text {SKP2 }}$ (Carrano et al., 1999; Sutterluty et al., 1999; Tsvetkov et al., 1999). In a SAG-transgenic mouse skin model, we found that p27 levels are much lower after UV exposure in SAG transgenic epidermis and primary keratinocytes, as compared to that in its non-transgenic littermates, whereas SAG silencing inhibits p27 ubiquitination, indicating SAG involvement in p27 degradation (He et al., 2008);

b) NOXA, a pro-apoptotic protein that promotes apoptosis via specifically inhibiting the anti-apoptotic Bcl-2 family member, Mcl-1 (Chen et al., 2005; Kim et al., 2006). It is totally unknown how NOXA is ubiquitinated and degraded. We found that SAG silencing causes significant NOXA accumulation by extending its protein half-life, whereas ectopic SAG expresssion significantly reduces NOXA levels by shortening its protein half-life (Jia et al., 2010). We further found that the small molecule MLN4924 causes a dosedpendent NOXA accumulation in multiple cancer cell lines tested (unpublished observation), indicating that NOXA is subjected to degradation by a SAG-CRL E3. Future study will be directed to identify the specific CRL responsible for NOXA degradation. It is truly worthy noting that MLN4924 is a small molecule inhibitor of Nedd8-Activating Enzyme, which inactivates CRL E3 ligase activity through cullin deneddylation (Soucy et al., 2009a; Brownell et al., 2010). MLN4924 is being developed as the first-in-class anti-cancer agent (Soucy et al., 2009a; Nawrocki et al., 2012), which suppresses tumor cell growth by effectively inducing cell death via apoptosis (Soucy et al., 2009a; Milhollen et al., 2010; Swords et al., 2010; Milhollen et al., 2011; Tan et al., 2011a; Zhao et al., 2011a), senescence (Lin et al., 2010a; Lin et al., 2010b; Jia et al., 2011) and autophagy (Luo et al., 2012; Zhao and Sun, 2012; Zhao et al., 2012). With promising preclinical efficacy, MLN4924 has been advanced to several Phase I 


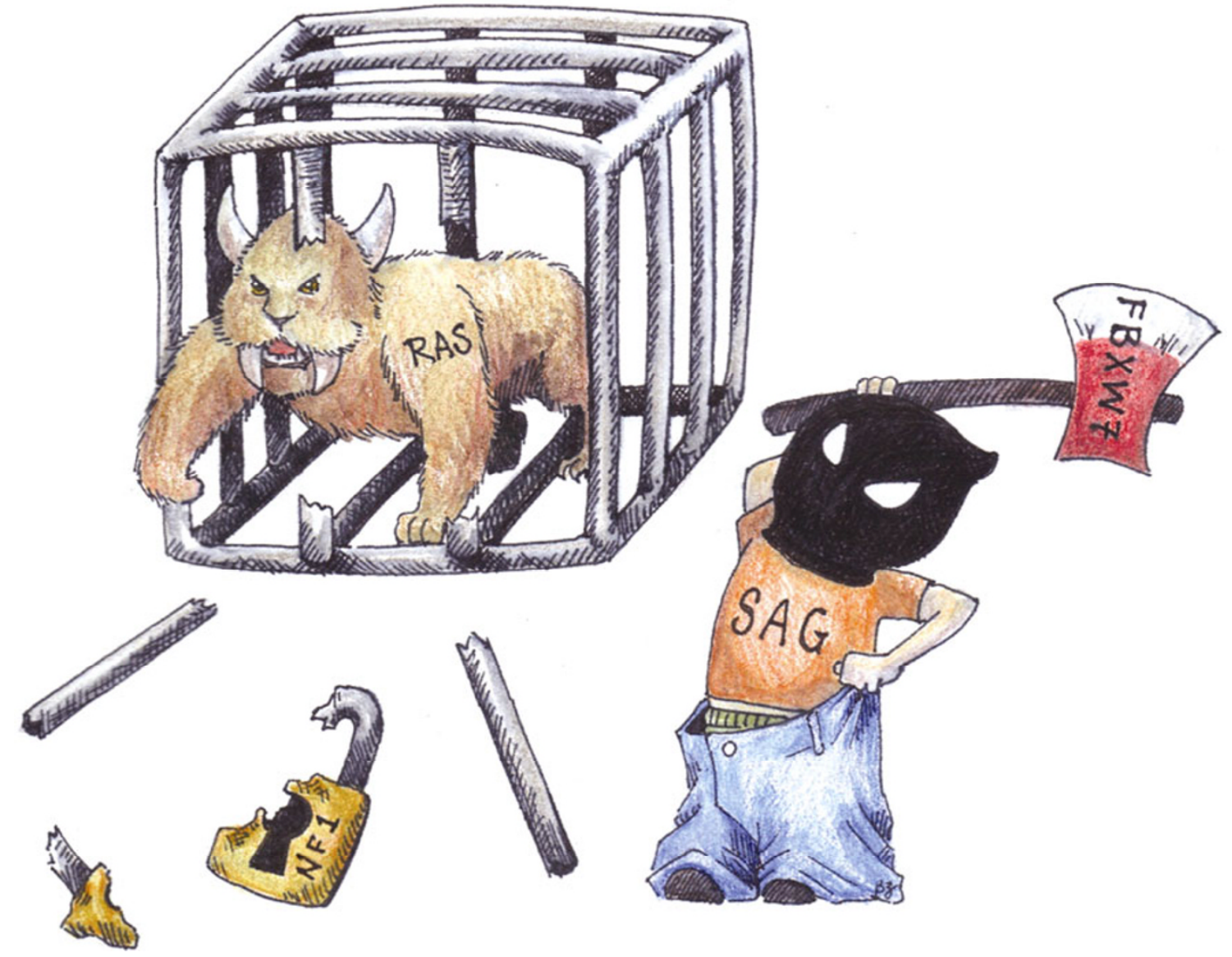

Figure 4. SAG E3 ligase activates RAS by degrading NF1. In response to different stimuli, SAG is induced and recruits FBXW7-CUL1 to form an active E3 ligase (man with an axe) that promotes the ubiquitination and degradation of NF1 (broken lock). Removal of NF1 releases its inhibitory effect on RAS, leading to RAS activation (monster out of cage) to promote cell proliferation, survival and oncogenesis. Thus, NF1 is a physiological substrate of SAG-FBXW7-CUL1 E3. Sag deletion induces NF1 accumulation to shut down RAS signals and causes the defects in vascular and neuronal development. (Image created by Becky Zhao and Steven Sun).

clinical trials against a number of human malignancies (Soucy et al., 2009b; Soucy et al., 2010).

c) DEPTOR, a newly identified naturally occurring inhibitor of mTORC1 and mTORC2 (Peterson et al., 2009). Most recently, we (Zhao et al., 2011b) and other two independent laboratories (Duan et al., 2011; Gao et al., 2011) found that DEPTOR is a physiological substrate of SCF ${ }^{\beta T r C P}$ E3 ligase [for Research Highlights, see (Wrighton, 2011)]. We found that MLN4924 causes DEPTOR accumulation in several cancer cell lines (Luo et al., 2012; Zhao et al., 2012), whereas ectopic SAG expression promotes DEPTOR degradation by $\mathrm{SCF}^{\beta \operatorname{TrCP}}$ E3 (unpublished observation). Thus, SAG-SCF is actively involved in DEPTOR ubiquitination and subsequent degradation.

\section{BIOLOGICAL FUNCTIONS}

\section{Apoptosis inhibition}

SAG was first cloned in our laboratory through the differential display (Liang and Pardee, 1992) as a redox inducible protein (Sun, 1997; Sun et al., 1997; Sun, 2000) that protects cancer cells from apoptosis induced by the redox compound 1,10-phenanthroline and zinc sulfate (Duan et al., 1999) in a RING domain dependent manner (Sun, 1999). Our subsequent studies showed in an in vivo ischemia mouse model that transduction of AdCMV-SAG (wild-type), but not its RING mutant, AdCMV-mSAG, nor the AdCMV-lacZ control, protects brain tissues from ischemic injury, as evidenced by significant reduction of the infarct areas where SAG was highly expressed. Mechanistic studies revealed that SAG overexpression decreases the production of ROS and reduces the number of apoptotic cells in the ischemic areas (Yang et al., 2001). Furthermore, using a cardiac myocyte ischemia model, we showed that overexpression of SAG, but not its RING mutant, protects cells from apoptotic death induced by simulated ischemia and reperfusion, whereas antisense SAG enhances cell death under both normoxic and hypoxic conditions. Thus, SAG is a cardio-protective protein in 
cardiac cells against ischemic stress (Chanalaris et al., 2003).

Subsequent studies, including our own, demonstrated in many cell culture models that SAG protects cells from apoptosis induced by nitric oxide (Yang and Park, 2006), heat shock (Lee et al., 2008), neurotoxins and 1-methyl-4-phenylpyridinium (Kim et al., 2007), UV-irradiation (He et al., 2008), ischemia-mediated oxidative stress (Kim et al., 2010; Yoo et al., 2012), and ionizing radiation (Kim et al., 2011). Consistently, SAG knockdown by anti-sense, siRNA or Sag knockout by gene deletion inhibits tumor cell growth (Huang et al., 2001), enhances apoptosis induced by etoposide and TRAIL (Tan et al., 2006), by actinomycin D and etoposide (Yang et al., 2010), by ionizing radiation (Tan et al., 2010; Kim et al., 2011) and by retinoic acid, which induces differentiation in wild type embryonic stem cells, but apoptosis upon Sag deletion (Tan et al., 2011a). These cellular functions are likely mediated by the combination of its antioxidant activity by scavenging ROS (Duan et al., 1999; Yang and Park, 2006; Lee et al., 2008; Kim et al., 2010; Tan et al., 2010; Kim et al., 2011; Yoo et al., 2012), and its E3 ubiquitin ligase activity by promoting the degradation of pro-caspase-3 (Tan et al., 2006), IkBa (Gu et al., 2007a; Tan et al., 2010), and NOXA (Jia et al., 2010) in a cell line and cell context dependent manner.

More significantly, we found that Sag total knockout causes embryonic lethality at embryonic stage of E11.5-12.5, which is associated with massive embryonic apoptosis (Tan et al., 2011b). The massive apoptosis seen in in vivo Sag ${ }^{-/}$ embryos is directly consistent with SAG's anti-apoptotic function observed in many in vitro cell culture studies (Duan et al., 1999; Sun et al., 2001) and firmly established that SAG is an anti-apoptotic protein.

\section{Radiation resistance}

We examined potential role of SAG in conferring cellular radioresistance, based upon two pieces of evidence. First, SAG is an antioxidant protein that scavenges ROS. ROS production is one of common mechanisms by which radiation induces cell killing (Cook et al., 2004). Second, SAG is an E3 ubiquitin ligase that promotes the ubiquitination and degradation of IKBa, leading to activation of NFKB (Gu et al., 2007a; Tan et al., 2010). NFKB is a survival transcription factor which often mediates adaptive radioresistance (Ahmed and $\mathrm{Li}$, 2008). Using both gene knockout (KO) and siRNA silencing approaches, we found that mouse ES cells with Sag KO are indeed much more sensitive to ionizing radiation with a sensitivity enhancement ratio (SER) of $\sim 1.6$. Mechanistically, enhanced radiation sensitivity is mediated by increased steady-state levels of intracellular ROS and decreased activation of NFKB (Tan et al., 2010). Consistently, SAG siRNA silencing in otherwise radioresistant human cancer cell lines causes radiosensitization (Jia et al., 2010). Furthermore, we recently showed that MLN4924, a small molecule inhibitor of SAG-SCF E3 ligase, sensitizes otherwise resistant pancreatic, lung, and breast cancer cells to ionizing radiation with a minimal effect on normal lung fibroblasts (Wei et al., 2012; Yang et al., 2012), suggesting that MLN4924 could act as a novel class of radiosensitizing agents (Wei, 2012). Taken together, these results indicate that SAG confers radioresistance and targeting SAG induces radiosensitivity.

\section{Mouse embryonic development}

In an attempt to understand the in vivo physiological function of SAG, we generated a Sag total KO mouse model through a gene trap approach and found that Sag is a developmentally essential gene whose disruption causes embryonic lethality at E11.5-12.5. The embryonic death is associated with overall growth retardation, massive apoptosis, and poor vasculogenesis (Tan et al., 2011b). The growth retardation and massive apoptosis seen in in vivo Sag ${ }^{-/}$embryos (Tan et al., 2011b) is consistent with SAG's role in promoting proliferation (Duan et al., 2001) and inhibiting apoptosis as seen in in vitro culture studies (Duan et al., 1999; Sun et al., 2001). Thus, despite Sag being dispensable during development in Drosophila and C. elegans (Moore and Boyd, 2004; Reynolds et al., 2008), we demonstrate that Sag is absolutely required for mouse development. The fact that Sag knockout in $R b x 1$ wild type background causes embryonic lethality at E11.5-12.5, whereas $R b x 1$ knockout in Sag wild type background also causes embryonic lethality but at even earlier stage of E7.5 (Tan et al., 2009), strongly indicates that Sag and its family member, $R b x 1$ are functionally non-redundant in mice, despite the fact that either SAG or RBX1 rescues yeast death upon targeted disruption of Hrt1, the only yeast homolog of RBX1/SAG (Ohta et al., 1999; Seol et al., 1999; Swaroop et al., 2000). Thus, although SAG and RBX1 are largely indistinguishable biochemically in promoting substrate ubiquitination, they are likely responsible for the degradation of different and non-overlapping sets of substrates to ensure a proper embryonic development.

\section{Vasculogenesis and angiogenesis}

Since the $\mathrm{Sag}^{-1-}$ embryos die at E11.5-12.5, we dissected the $\mathrm{Sag}^{-/-}$embryos at E10.5 and found a remarkable reduction of vasculogenesis in both yolk-sacs and embryonic brains (Tan et al., 2011b). To define the mechanism, we established Sag ${ }^{-1-}$ embryonic stem cells (ESCs) and determined the ability of $\mathrm{Sag}^{-1-}$ ESCs to undergo endothelial differentiation, an in vitro assay mimicking the in vivo vasculogenesis. We found that Sag ${ }^{-1}$ ESCs are defective in endothelial differentiation and completely fail to form cystic embryoid bodies containing blood island structures, although they are able to differentiate to form embryoid bodies (Tan et al., 2011b). The Sag ${ }^{-1-}$ ES cells also have a significantly reduced rate of formation of teratoma when injected s.c into nude mice. Immunostaining of teratoma tissues derived from $\mathrm{Sag}^{-1-}$ ES cells shows significant reduction in vessel density and rate of cell proliferation. Further mechanistic studies 
revealed that Sag deletion caused accumulation of Nf1 tumor suppressor, a naturally occurring inhibitor of RAS. Importantly, the defect in endothelial differentiation of $\mathrm{Sag}^{-1}$ ES cells can be fully rescued by simultaneous deletion of even one allele of Nf1, which is sufficient to reactivate RAS-MAPK pathways, indicating that accumulated Nf1 plays a causal role (Tan et al., 2011b) (Fig. 5). More significantly, we found that simultaneous

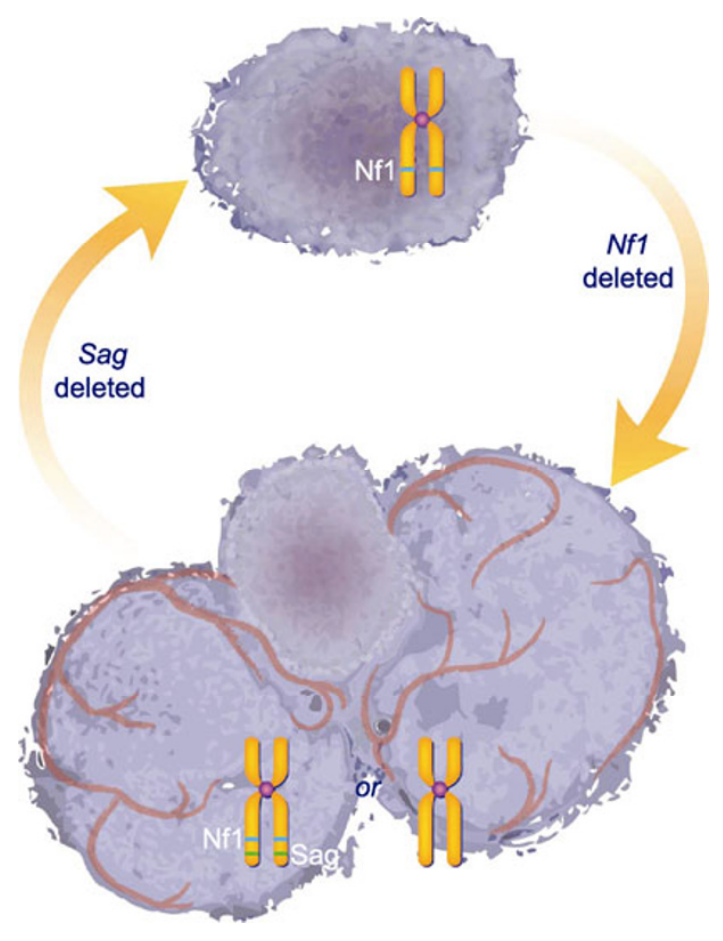

Figure 5. Sag/Nf1 regulates vasculogenesis. Under suspension culture in the absence of leukemia inhibitory factor, wild type mouse embryonic stem cells undergo endothelial differentiation to form cystic embryoid bodies with blood vessel structure (bottom). Sag deletion completely blocks endothelial differentiation and abrogates the formation of cystic embryoid bodies (top). Mechanistic studies revealed that tumor suppressor Nf1 is a physiological substrate of Sag-Fbxw7-Cul1 E3. Sag deletion causes Nf1 accumulation to inhibit Ras and blocks MAPK signals. Simultaneous deletion of Sag and Nf1 completely rescues the defect of Sag deletion in endothelial differentiation and restores the formation of cystic embryoid bodies (bottom). (Image created by Steven Kronenberg).

deletion of Nf1 and Sag can partially rescue vascular defects seen in Sag ${ }^{-1-}$ embryos (Tan et al., 2011b). We performed detailed biochemical characterization of SAG-NF1 interaction and showed that SAG-SCF ${ }^{\text {FBXW7 }}$ E3 ligase targets NF1 for ubiquitination and degradation (Tan et al., 2011b). Thus, Nf1, as a physiological substrate of Sag, accumulates upon Sag deletion to inhibit RAS and abrogate RAS-MAPK signaling pathways, leading to blockage of proliferation and endothelial differentiation in vitro and growth retardation and poor vasculogensis in vivo (Figs. 4 and 5). Given the fact that Sag-SCF E3 targets for degradation many cellular substrates which play the essential roles in embryogenesis (Wei and Sun, 2010), it is expected that deletion of accumulated Nf1 alone is not sufficient to cause a full rescue of embryonic lethality.

To further study the role of Sag in regulation of vasculogenesis, we have recently generated Sag $^{f / f l}$ conditional mice and found endothelial cells from Sag ${ }^{f / f l}$ mice have a significantly reduced capacity for cell migration upon Ad-Cre-mediated Sag deletion. More importantly, angiogenesis induced by tumor cells is also significantly reduced in Sag $^{\text {fl/fl }}$ mice (unpublished observations). Thus, Sag is required for proper vasculogenesis during embryogenesis and angiogenesis in adult mouse.

\section{Tumorigenesis}

The potential role that Sag may play in tumorigenesis is suggested by the observations that a) SAG is overexpressed in carcinomas of lung, colon, stomach and liver (Huang et al., 2001; Jia et al., 2010), b) SAG overexpression is associated with poor prognosis of patients with lung cancer (Sasaki et al., 2001) and rectal cancer (Ozden et al., 2011), and c) SAG promotes cell growth under serum starved stress condition and microinjection of SAG mRNA into quiescent NIH3T3 cells induces S phase entry (Duan et al., 2001); SAG also promotes cell proliferation in mouse hippocampal dentate gyrus (Yoo et al., 2012). To address SAG's role in tumorigenesis, we established a K14-SAG transgenic (SAG-Tg) mouse model in which SAG expression in skin epidermis is driven by the K14 promoter. In a DMBA/TPA two-stage skin caricinogenesis study, we found SAG skin transgenic expression inhibited tumor formation at the early stage by targeting c-Jun for degradation to inactivate AP-1, but enhanced tumor growth at the later stage by targeting IкBa for degradation to activate NFKB (Gu et al., 2007a). The net outcome of this stage-dependent targeting of different SAG substrates (c-Jun vs. IKBa) is that SAG-Tg develops fewer skin papillomas due to reduced AP-1 promotion, but papillomas that did develop grow much bigger due to reduced apoptosis as result of NFKB activation (Gu et al., 2007a). In a UVB skin carcinogenesis model, SAG skin transgenic expression targets both c-Jun and p27 for degradation, leading to reduced c-Jun level and AP-1 activity as well as reduced p27 level. The net outcome of SAG-mediated c-Jun/AP-1 inactivation and p27 reduction is an increase in the development of skin hyperplasia, as evidenced by increased skin thickness and increased rate of DNA synthesis, but not skin tumors (He et al., 2008). Thus, compared to c-Jun/AP-1, p27 appears to plays a bigger role in regulation of UVB-induced skin proliferation. Finally, we have extended this in vivo tumorigenesis study to other mouse models. Our newly generated unpublished data showed that Sag is required for Kras-induced lung tumorigenesis, as tested in a well-established $\mathrm{Kras}^{\mathrm{G} 12 \mathrm{D}}$ lung tumorigenesis model (Jackson et al., 2001). 


\section{CONCLUSION AND FUTURE PERSPECTIVES}

In summary, the data reviewed here support the following model. In response to various internal and external stimuli, such as hypoxia and ROS, SAG is induced as a cellular protective mechanism against oxidative stress. Induced SAG can act alone as an antioxidant to scavenge ROS, thus blocking ROS-induced DNA damage, lipid peroxidation, and apoptosis (Fig. 6, left panel). Induced SAG also recruits other components of SCF E3 and promotes the ubiquitination and subsequent degradation of a) NOXA and pro-caspase-3 to block apoptosis, b) IkBa to activate NFKB, c) p27 to activate CDKs (cyclin-dependent kinases), d) NF1 to activate RAS, and e) DEPTOR to activate mTORCs, leading to apoptosis inhibition, radioresistance, enhanced proliferation and angiogenesis, and accelerated tumorigenesis (Fig. 6, right panel). Targeting SAG via genetic (siRNA silencing or gene deletion) or pharmaceutical (MLN4924) approaches reverses these oncogenic processes, Thus, SAG is an attractive anti-cancer and radiosensitizing target (Sun, 2003, 2006; Wei and Sun, 2010; Jia and Sun, 2011).

\section{Several future directions around SAG study are articulated below}

First of all, we need to biochemically and functionally differentiate SAG from its family member RBX1. It has been previously reported that $S A G$ prefers to form the complex with cullin-5-SOCS-box proteins, whereas RBX1 prefers with cullin-2-VHL-box (Kamura et al., 2004; Kohroki et al., 2005). However, we do observe SAG involvement in ubiquitination and degradation of IKB, NF1, procaspase-3 and DEPTOR (cullin-1 mediated) (Tan et al., 2006; Gu et al., 2007a; Tan et al., 2010, 2011b) and HIF-1a (cullin-2 mediated) (Tan et al., 2008), indicating that SAG could also form the complex with other CRLs; This study may shed light on why SAG and RBX1 function non-redundantly during mouse embryogenesis.

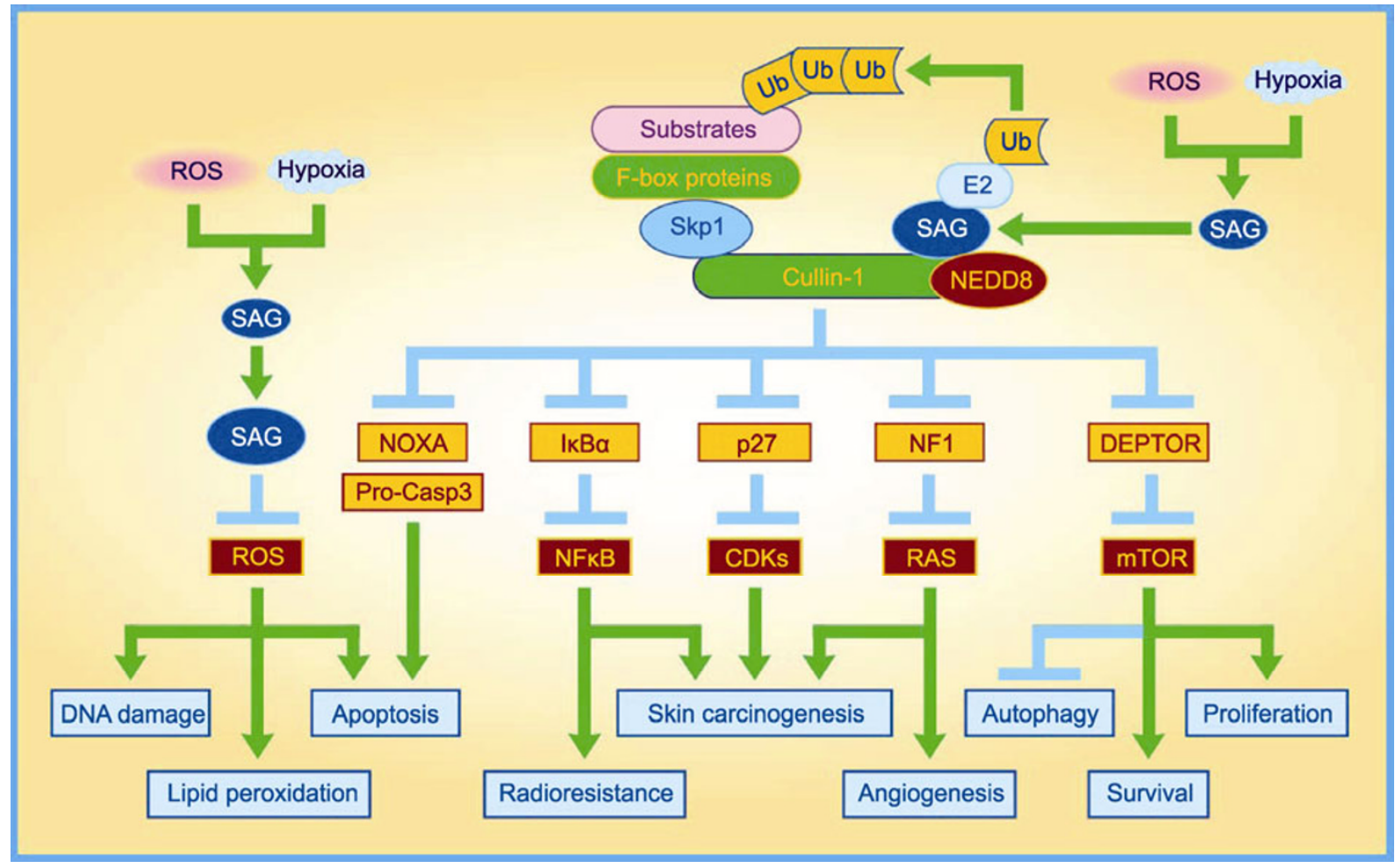

Figure 6. SAG regulates multiple biological processes. SAG is induced in response to stresses such as hypoxia and ROS. Induced SAG scavenges ROS by acting alone as an antioxidant (left panel) or promotes ubiquitination and degradation of tumor suppressive substrates by forming active SAG-SCF E3 ubiquitin ligase, leading to apoptosis protection and tumorigenesis (right panel, see text for details).

Secondly, we need to identify and characterize endogenous and physiologically relevant substrates of SAG-Cul5SOCS E3, an area in which the research progress is relatively lacking. A recent study showed that Elongin $A / B / C$ complex assembles with SAG/Cul5 to form an active E3 to promote ubiquitination and degradation of Rpb1, the largest subunit of pol II (Yasukawa et al., 2008). In other two cases, although active Src oncoprotein and Dab1, an intracellular signaling molecule, appear to subject to degradation by CRL5, potential involvement of SAG is unknown (Feng et al., 2007; Laszlo and Cooper, 2009; Simo et al., 2010; Pan et al., 2011). Still unclear is the active role of SAG-Cul-5 in SOCS2-mediated degradation of filamin B (Bello et al., 2009), iNOS (Kuang et al., 2009), and growth hormone receptor 
(Vesterlund et al., 2011). On the other hand, it has been relatively well established that several viral proteins can hijack cellular cullin-5 E3 to promote degradation of key host proteins, such as anti-viral protein APOBEC3G (Yu et al., 2003), tumor suppressors p53 (Querido et al., 2001) and VHL (Cai et al., 2006); the SAG involvement is again totally unknown. Our understanding of how SAG-Cul-5 E3 regulates viral infection may lead to a novel design of anti-viral therapy surrounding this E3 ligase.

Thirdly, we need to understand how SAG is regulated at the post-translational level. We know that SAG is subjected to transcriptional activation by AP-1 (Gu et al., 2007b) and HIF-1 (Tan et al., 2008), and we also know that SAG is subjected to ubiquitination and degradation (unpublished observation). However, we do not know how SAG protein stability is regulated by which E3 ligase and deubiquitinase, although NEDD4 E3 has been shown to bind with SAG/RNF7 in a proteome array study (Persaud et al., 2009). Given the fact that SAG is an attractive target, a novel anticancer approach may involve the discovery of small molecule inhibitors of SAG deubiquitinase, which would block SAG deubiquitination, leading to an enhanced SAG degradation.

Finally, we need to achieve a better understating of SAG's role in tumorigenesis. We know that SAG is not a dominant oncogene in our skin transgenic model (Gu et al., 2007a), but it could be an oncogenic-cooperative gene that is required for tumorigenesis driven by activation of oncogene(s) or deletion of tumor suppressor gene(s). Availability of the Sag conditional KO mouse model ( $\mathrm{Sag}^{f / f l}$ mouse) makes it possible to address this important question. Better understanding of these research questions will lay a solid foundation for the future discovery of SAG E3 specific inhibitors against human cancers with SAG overexpression and activation.

\section{ACKNOWLEDGEMENTS}

This work was supported by the $\mathrm{NCl}$ grants (CA118762 and CA156744) to Yi Sun.

\section{ABBREVIATIONS}

CK2, caseine kinase II; CRL, Cullin-RING ligase; DTT, dithiothreitol; ESC, embryonic stem cell; GAP, GTPase activating protein; HECT, homology to E6AP C-terminus; NEM, N-ethylmaleimide; RBX2, RING box protein 2; RING, really interesting new gene; RNF7, RING finger protein 7; ROC2, regulator of cullins-2; SAG, sensitive to apoptosis gene; SCF, SKP1-cullin 1-F-box protein; SIN-1, 3-morphol-inosydnomine $\mathrm{N}$-ethylcarbamide

\section{REFERENCES}

Ahmed, K.M., and Li, J.J. (2008). NF-kappa B-mediated adaptive resistance to ionizing radiation. Free Radic Biol Med 44, 1-13.

Angel, P., and Karin, M. (1991). The role of Jun, Fos and the AP-1 complex in cell-proliferation and transformation. Biochim Biophys Acta 1072, 129-157.

Baeuerle, P.A., and Baltimore, D. (1988a). Activation of DNA-binding activity in an apparently cytoplasmic precursor of the NF-kappa B transcription factor. Cell 53, 211-217.

Baeuerle, P.A., and Baltimore, D. (1988b). I kappa B: a specific inhibitor of the NF-kappa B transcription factor. Science 242, 540-546.

Bello, N.F., Lamsoul, I., Heuze, M.L., Metais, A., Moreaux, G., Calderwood, D.A., Duprez, D., Moog-Lutz, C., and Lutz, P.G. (2009). The E3 ubiquitin ligase specificity subunit ASB2beta is a novel regulator of muscle differentiation that targets filamin $B$ to proteasomal degradation. Cell Death Differ 16, 921-932.

Brownell, J.E., Sintchak, M.D., Gavin, J.M., Liao, H., Bruzzese, F.J., Bump, N.J., Soucy, T.A., Milhollen, M.A., Yang, X., Burkhardt, A.L., et al. (2010). Substrate-assisted inhibition of ubiquitin-like protein-activating enzymes: the NEDD8 E1 inhibitor MLN4924 forms a NEDD8-AMP mimetic in situ. Mol Cell 37, 102-111.

Cai, Q.L., Knight, J.S., Verma, S.C., Zald, P., and Robertson, E.S. (2006). EC5S ubiquitin complex is recruited by KSHV latent antigen LANA for degradation of the VHL and p53 tumor suppressors. PLoS Pathog 2, e116.

Carrano, A.C., Eytan, E., Hershko, A., and Pagano, M. (1999). SKP2 is required for ubiquitin-mediated degradation of the CDK inhibitor p27. Nat Cell Biol 1, 193-199.

Chanalaris, A., Sun, Y., Latchman, D.S., and Stephanou, A. (2003). SAG attenuates apoptotic cell death caused by simulated ischaemia/reoxygenation in rat cardiomyocytes. J Mol Cell Cardiol 35, 257-264.

Chen, L., Willis, S.N., Wei, A., Smith, B.J., Fletcher, J.I., Hinds, M.G., Colman, P.M., Day, C.L., Adams, J.M., and Huang, D.C. (2005). Differential targeting of prosurvival $\mathrm{Bcl}-2$ proteins by their BH3-only ligands allows complementary apoptotic function. Mol Cell 17, 393-403.

Cichowski, K., and Jacks, T. (2001). NF1 tumor suppressor gene function: narrowing the GAP. Cell 104, 593-604.

Ciechanover, A. (1998). The ubiquitin-proteasome pathway: on protein death and cell life. EMBO J 17, 7151-7160.

Cook, J.A., Gius, D., Wink, D.A., Krishna, M.C., Russo, A., and Mitchell, J.B. (2004). Oxidative stress, redox, and the tumor microenvironment. Semin Radiat Oncol 14, 259-266.

Cryns, V., and Yuan, J. (1998). Proteases to die for. Genes Dev 12, 1551-1570.

Deneke, S.M. (2000). Thiol-based antioxidants. Curr Top Cell Regul 36, 151-180.

Deshaies, R.J., and Joazeiro, C.A. (2009). RING domain E3 ubiquitin ligases. Annu Rev Biochem 78, 399-434.

Dhillon, A.S., Hagan, S., Rath, O., and Kolch, W. (2007). MAP kinase signalling pathways in cancer. Oncogene 26, 3279-3290.

Duan, H., Tsvetkov, L.M., Liu, Y., Song, Y., Swaroop, M., Wen, R., Kung, H.F., Zhang, H., and Sun, Y. (2001). Promotion of S-phase entry and cell growth under serum starvation by SAG/ROC2/Rbx2/Hrt2, an E3 ubiquitin ligase component: association with inhibition of p27 accumulation. Mol Carcinog 30 , 37-46.

Duan, H., Wang, Y., Aviram , M., Swaroop, M., Loo, J.A., Bian, J., Tian, Y., Mueller, T., Bisgaier, C.L., and Sun, Y. (1999). SAG, a novel zinc RING finger protein that protects cells from apoptosis induced by redox agents. Mol Cell Biol 19, 3145-3155.

Duan, S., Skaar, J.R., Kuchay, S., Toschi, A., Kanarek, N., Ben-Neriah, Y., and Pagano, M. (2011). mTOR Generates an 
auto-amplification loop by triggering the betaTrCP- and CK1alpha-dependent degradation of DEPTOR. Mol Cell 44, 317-324.

Earnshaw, W.C., Martins, L.M., and Kaufmann, S.H. (1999). Mammalian caspases: structure, activation, substrates, and functions during apoptosis. Annu Rev Biochem 68, 383-424.

Feng, L., Allen, N.S., Simo, S., and Cooper, J.A. (2007). Cullin 5 regulates Dab1 protein levels and neuron positioning during cortical development. Genes Dev 21, 2717-2730.

Fenner, B.J., Scannell, M., and Prehn, J.H. (2010). Expanding the substantial interactome of NEMO using protein microarrays. PLoS One 5, e8799.

Frei, B., Stocker, R., and Ames, B.N. (1988). Antioxidant defenses and lipid peroxidation in human blood plasma. Proc Natl Acad Sci U S A 85, 9748-9752.

Fuchs, S.Y., Chen, A., Xiong, Y., Pan, Z.Q., and Ronai, Z. (1999). HOS, a human homolog of Slimb, forms an SCF complex with Skp1 and Cullin1 and targets the phosphorylation-dependent degradation of IkappaB and beta-catenin. Oncogene 18, 2039-2046.

Gao, D., Inuzuka, H., Tan, M.K., Fukushima, H., Locasale, J.W., Liu, P., Wan, L., Zhai, B., Chin, Y.R., Shaik, S., et al. (2011). mTOR drives its own activation via $\operatorname{SCF}($ betaTrCP)-dependent degradation of the mTOR inhibitor DEPTOR. Mol Cell 44, 290-303.

Gu, Q., Bowden, G.T., Normolle, D., and Sun, Y. (2007a). SAG/ROC2 E3 ligase regulates skin carcinogenesis by stage-dependent targeting of c-Jun/AP1 and IkappaB-alpha/ NF-kappaB. J Cell Biol 178, 1009-1023.

Gu, Q., Tan, M., and Sun, Y. (2007b). SAG/ROC2/Rbx2 is a novel activator protein-1 target that promotes c-Jun degradation and inhibits 12-O-tetradecanoylphorbol-13-acetate-induced neoplastic transformation. Cancer Res 67, 3616-3625.

He, H., Gu, Q., Zheng, M., Normolle, D., and Sun, Y. (2008). SAG/ROC2/RBX2 E3 ligase promotes UVB-induced skin hyperplasia, but not skin tumors, by simultaneously targeting c-Jun/AP-1 and p27. Carcinogenesis 29, 858-865.

He, H., Tan, M., Pamarthy, D., Wang, G., Ahmed, K., and Sun, Y. (2007). CK2 phosphorylation of SAG at Thr10 regulates SAG stability, but not its E3 ligase activity. Mol Cell Biochem 295, 179-188.

Hershko, A., and Ciechanover, A. (1998). The ubiquitin system. Annu Rev Biochem 67, 425-479.

Hershko, A., Ciechanover, A., and Varshavsky, A. (2000). Basic Medical Research Award. The ubiquitin system. Nat Med 6, 1073-1081.

Huang, Y., Duan, H., and Sun, Y. (2001). Elevated expression of $\mathrm{SAG} / \mathrm{ROC2} / \mathrm{Rb} 2 / \mathrm{Hrt} 2$ in human colon carcinomas: SAG does not induce neoplastic transformation, but its antisense transfection inhibits tumor cell growth. Mol Carcinog 30, 62-70.

Ivan, M., Kondo, K., Yang, H., Kim, W., Valiando, J., Ohh, M., Salic, A., Asara, J.M., Lane, W.S., and Kaelin, W.G., Jr. (2001). HIFalpha targeted for VHL-mediated destruction by proline hydroxylation: implications for O2 sensing. Science 292, 464-468.

Jaakkola, P., Mole, D.R., Tian, Y.M., Wilson, M.I., Gielbert, J., Gaskell, S.J., Kriegsheim, A., Hebestreit, H.F., Mukherji, M., Schofield, C.J., et al. (2001). Targeting of HIF-alpha to the von
Hippel-Lindau ubiquitylation complex by O2-regulated prolyl hydroxylation. Science 292, 468-472.

Jackson, E.L., Willis, N., Mercer, K., Bronson, R.T., Crowley, D., Montoya, R., Jacks, T., and Tuveson, D.A. (2001). Analysis of lung tumor initiation and progression using conditional expression of oncogenic K-ras. Genes Dev 15, 3243-3248.

Jia, L., Li, H., and Sun, Y. (2011). Induction of p21-Dependent Senescence by an NAE Inhibitor, MLN4924, as a Mechanism of Growth Suppression. Neoplasia 13, 561-569.

Jia, L., and Sun, Y. (2011). SCF E3 ubiquitin ligases as anticancer targets. Curr Cancer Drug Targets 11, 347-356.

Jia, L., Yang, J., Hao, X., Zheng, M., He, H., Xiong, X., Xu, L., and Sun, Y. (2010). Validation of SAG/RBX2/ROC2 E3 Ubiquitin Ligase as an Anticancer and Radiosensitizing Target. Clin Cancer Res 16, 814-824.

Jin, J., Cardozo, T., Lovering, R.C., Elledge, S.J., Pagano, M., and Harper, J.W. (2004). Systematic analysis and nomenclature of mammalian F-box proteins. Genes Dev 18, 2573-2580.

Kamura, T., Maenaka, K., Kotoshiba, S., Matsumoto, M., Kohda, D., Conaway, R.C., Conaway, J.W., and Nakayama, K.I. (2004). VHL-box and SOCS-box domains determine binding specificity for Cul2-Rbx1 and Cul5-Rbx2 modules of ubiquitin ligases. Genes Dev 18, 3055-3065.

Kim, D.W., Lee, S.H., Jeong, M.S., Sohn, E.J., Kim, M.J., Jeong, H.J., An, J.J., Jang, S.H., Won, M.H., Hwang, I.K., et al. (2010). Transduced Tat-SAG fusion protein protects against oxidative stress and brain ischemic insult. Free Radic Biol Med 48, 969-977.

Kim, H., Rafiuddin-Shah, M., Tu, H.C., Jeffers, J.R., Zambetti, G.P., Hsieh, J.J., and Cheng, E.H. (2006). Hierarchical regulation of mitochondrion-dependent apoptosis by BCL-2 subfamilies. Nat Cell Biol 8, 1348-1358.

Kim, S.Y., Bae, Y. S., Park, J. W. (2002). Thio-linked peroxidase activity of human sensitive to apoptosis gene (SAG) protein. Free Radic Res 36, 73-78.

Kim, S.Y., Kim, M.Y., Mo, J.S., Park, J.W., and Park, H.S. (2007). SAG protects human neuroblastoma SH-SY5Y cells against 1-methyl-4-phenylpyridinium ion (MPP+)-induced cytotoxicity via the downregulation of ROS generation and JNK signaling. Neurosci Lett 413, 132-136.

Kim, S.Y., Lee, J. H., Yang, E. S., Kil, I. S., Bae, Y. S. (2003a). Human sensitive to apoptosis gene protein inhibits peroxynitrite-induced DNA damage. Biochem Biophys Res Commun 301, 671-674.

Kim, S.Y., Yang, E.S., Lee, Y.S., Lee, J., and Park, J.W. (2011). Sensitive to apoptosis gene protein regulates ionizing radiation-induced apoptosis. Biochimie 93, 269-276.

Kim, Y.S., Lee, J. Y., Son, M. Y., Park, W., Bae, Y.S. (2003b). Phosphorylation of threonine-10 on CKBBP1/SAG/ROC2/Rbx2 by protein kinase CKII promotes the degradation of $\mathrm{IkBa}$ and p2 $7^{\text {kip1 }}$. J. Biol. Chem 278, 28462-28469.

Kohroki, J., Nishiyama, T., Nakamura, T., and Masuho, Y. (2005). ASB proteins interact with Cullin5 and Rbx2 to form E3 ubiquitin ligase complexes. FEBS Lett 579, 6796-6802.

Kranenburg, O., Gebbink, M.F., and Voest, E.E. (2004). Stimulation of angiogenesis by Ras proteins. Biochim Biophys Acta 1654, 23-37. 
Kuang, Z., Yao, S., Xu, Y., Lewis, R.S., Low, A., Masters, S.L., Willson, T.A., Kolesnik, T.B., Nicholson, S.E., Garrett, T.J., et al. (2009). SPRY domain-containing SOCS box protein 2: crystal structure and residues critical for protein binding. $\mathrm{J} \mathrm{Mol} \mathrm{Biol} \mathrm{386,}$ 662-674.

Laszlo, G.S., and Cooper, J.A. (2009). Restriction of Src activity by Cullin-5. Curr Biol 19, 157-162.

Le, L.Q., and Parada, L.F. (2007). Tumor microenvironment and neurofibromatosis type I: connecting the GAPs. Oncogene 26, 4609-4616.

Lee, J., and Zhou, P. (2010). Cullins and cancer. Genes Cancer 1, 690-699.

Lee, S.J., Yang, E.S., Kim, S.Y., Shin, S.W., and Park, J.W. (2008). Regulation of heat shock-induced apoptosis by sensitive to apoptosis gene protein. Free Radic Biol Med 45, 167-176.

Li, W., Bengtson, M.H., Ulbrich, A., Matsuda, A., Reddy, V.A., Orth, A., Chanda, S.K., Batalov, S., and Joazeiro, C.A. (2008). Genome-wide and functional annotation of human E3 ubiquitin ligases identifies MULAN, a mitochondrial E3 that regulates the organelle's dynamics and signaling. PLoS One 3, e1487.

Liang, P., and Pardee, A.B. (1992). Differential display of eukaryotic messenger RNA by means of the polymerase chain reaction [see comments]. Science 257, 967-971.

Lin, H.K., Chen, Z., Wang, G., Nardella, C., Lee, S.W., Chan, C.H., Yang, W.L., Wang, J., Egia, A., Nakayama, K.I., et al. (2010a). Skp2 targeting suppresses tumorigenesis by Arf-p53-independent cellular senescence. Nature 464, 374-379.

Lin, J.J., Milhollen, M.A., Smith, P.G., Narayanan, U., and Dutta, A. (2010b). NEDD8-targeting drug MLN4924 elicits DNA rereplication by stabilizing Cdt1 in S phase, triggering checkpoint activation, apoptosis, and senescence in cancer cells. Cancer Res 70, 10310-10320.

Lipkowitz, S., and Weissman, A.M. (2011). RINGs of good and evil: RING finger ubiquitin ligases at the crossroads of tumour suppression and oncogenesis. Nat Rev Cancer 11, 629-643.

Luo, Z., Yu, G., Lee, H.W., Li, L., Wang, L., Yang, D., Pan, Y., Ding, C., Qian, J., Wu, L., et al. (2012). The Nedd8-activating enzyme inhibitor MLN4924 induces autophagy and apoptosis to suppress liver cancer cell growth. Cancer Res 72, 3360-3371.

Maxwell, P.H., Wiesener, M.S., Chang, G.W., Clifford, S.C., Vaux, E.C., Cockman, M.E., Wykoff, C.C., Pugh, C.W., Maher, E.R., and Ratcliffe, P.J. (1999). The tumour suppressor protein VHL targets hypoxia-inducible factors for oxygen-dependent proteolysis. Nature 399, 271-275.

Milhollen, M.A., Narayanan, U., Soucy, T.A., Veiby, P.O., Smith, P.G., and Amidon, B. (2011). Inhibition of NEDD8-activating enzyme induces rereplication and apoptosis in human tumor cells consistent with deregulating CDT1 turnover. Cancer Res 71, 3042-3051.

Milhollen, M.A., Traore, T., Adams-Duffy, J., Thomas, M.P., Berger, A.J., Dang, L., Dick, L.R., Garnsey, J.J., Koenig, E., Langston, S.P., et al. (2010). MLN4924, a NEDD8-activating enzyme inhibitor, is active in diffuse large B-cell lymphoma models: rationale for treatment of NF-\{kappa\}B-dependent lymphoma. Blood 116, 1515-1523.

Moore, R., and Boyd, L. (2004). Analysis of RING finger genes required for embryogenesis in C. elegans. Genesis $38,1-12$.
Nakayama, K.I., and Nakayama, K. (2006). Ubiquitin ligases: cell-cycle control and cancer. Nat Rev Cancer 6, 369-381.

Nateri, A.S., Riera-Sans, L., Da Costa, C., and Behrens, A. (2004). The ubiquitin ligase SCFFbw7 antagonizes apoptotic JNK signaling. Science 303, 1374-1378.

Nawrocki, S.T., Griffin, P., Kelly, K.R., and Carew, J.S. (2012). MLN4924 : a novel first-in-class inhibitor of NEDD8-activating enzyme for cancer therapy. Expert Opin Investig Drugs 21, 1563-1573.

Ohta, T., Michel, J.J., Schottelius, A.J., and Xiong, Y. (1999). ROC1, a homolog of APC11, represents a family of cullin partners with an associated ubiquitin ligase activity. Mol Cell 3, 535-541.

Ozden, S.A., Ozyurt, H., Ozgen, Z., Kilinc, O., Oncel, M., Gul, A.E., Karadayi, N., Serakinci, N., Kan, B., and Orun, O. (2011). Prognostic role of sensitive-to-apoptosis gene expression in rectal cancer. World J Gastroenterol 17, 4905-4910.

Pan, Q., Qiao, F., Gao, C., Norman, B., Optican, L., and Zelenka, P.S. (2011). Cdk5 targets active Src for ubiquitin-dependent degradation by phosphorylating Src(S75). Cell Mol Life Sci 68, 3425-3436.

Persaud, A., Alberts, P., Amsen, E.M., Xiong, X., Wasmuth, J., Saadon, Z., Fladd, C., Parkinson, J., and Rotin, D. (2009). Comparison of substrate specificity of the ubiquitin ligases Nedd4 and Nedd4-2 using proteome arrays. Mol Syst Biol 5, 333.

Peterson, T.R., Laplante, M., Thoreen, C.C., Sancak, Y., Kang, S.A., Kuehl, W.M., Gray, N.S., and Sabatini, D.M. (2009). DEPTOR is an mTOR inhibitor frequently overexpressed in multiple myeloma cells and required for their survival. Cell 137, 873-886.

Querido, E., Blanchette, P., Yan, Q., Kamura, T., Morrison, M., Boivin, D., Kaelin, W.G., Conaway, R.C., Conaway, J.W., and Branton, P.E. (2001). Degradation of p53 by adenovirus E4orf6 and E1B55K proteins occurs via a novel mechanism involving a Cullin-containing complex. Genes Dev 15, 3104-3117.

Reynolds, P.J., Simms, J.R., and Duronio, R.J. (2008). Identifying determinants of cullin binding specificity among the three functionally different Drosophila melanogaster Roc proteins via domain swapping. PLoS One 3, e2918.

Sarikas, A., Hartmann, T., and Pan, Z.Q. (2011). The cullin protein family. Genome Biol 12, 220.

Sasaki, H., Yukiue, H., Kobayashi, Y., Moriyama, S., Nakashima, Y., Kaji, M., Fukai, I., Kiriyama, M., Yamakawa, Y., and Fujii, Y. (2001). Expression of the sensitive to apoptosis gene, SAG, as a prognostic marker in nonsmall cell lung cancer. Int J Cancer 95, 375-377.

Sato, M., and Bremner, I. (1993). Oxygen free radicals and metallothionein. Free Radic Biol Med 14, 325-337.

Semenza, G.L. (2003). Targeting HIF-1 for cancer therapy. Nat Rev Cancer 3, 721-732.

Seol, J.H., Feldman, R.M.R., Zachariae, W.Z., Shevchenko, A., Correll, C.C., Lyapina, S., Chi, Y., Galova, M., Claypool, J., Sandmeyer, S., et al. (1999). Cdc53/cullin and the essential Hrt1 RING-H2 subunit of SCF define a ubiquitin ligase module that activates the E2 enzyme Cdc34. Genes \& Dev 13, 1614-1626.

Shaulian, E., and Karin, M. (2002). AP-1 as a regulator of cell life and death. Nat Cell Biol 4, E131-136.

Sherr, C.J., and Roberts, J.M. (1995). Inhibitors of mammalian G1 cyclin-dependent kinases. Genes Dev 9, 1149-1163. 
Sherr, C.J., and Roberts, J.M. (1999). CDK inhibitors: positive and negative regulators of G1-phase progression. Genes Dev 13, 1501-1512.

Simo, S., Jossin, Y., and Cooper, J.A. (2010). Cullin 5 regulates cortical layering by modulating the speed and duration of Dab1-dependent neuronal migration. J Neurosci 30, 5668-5676.

Son, M.Y., Park, J.W., Kim, Y.S., Kang, S.W., Marshak, D.R., Park, W., and Bae, Y.S. (1999). Protein kinase CKII interacts with and phosphorylates the SAG protein containing ring- $\mathrm{H} 2$ finger motif. Biochem Biophys Res Commun 263, 743-748.

Soucy, T.A., Dick, L.R., Smith, P.G., Milhollen, M.A., and Brownell, J.E. (2010). The NEDD8 conjugation pathway and its relevance in cancer biology and therapy. Genes Cancer 1, 708-716.

Soucy, T.A., Smith, P.G., Milhollen, M.A., Berger, A.J., Gavin, J.M., Adhikari, S., Brownell, J.E., Burke, K.E., Cardin, D.P., Critchley, S., et al. (2009a). An inhibitor of NEDD8-activating enzyme as a new approach to treat cancer. Nature 458, 732-736.

Soucy, T.A., Smith, P.G., and Rolfe, M. (2009b). Targeting NEDD8-activated cullin-RING ligases for the treatment of cancer. Clin Cancer Res 15, 3912-3916.

Sun, Y. (1990). Free radicals, antioxidant enzymes, and carcinogenesis. Free Radic Biol Med 8, 583-599.

Sun, Y. (1997). Induction of glutathione synthetase by 1,10-phenanthroline. FEBS Lett 408, 16-20.

Sun, Y. (1999). Alteration of SAG mRNA in human cancer cell lines: requirement for the RING finger domain for apoptosis protection. Carcinogenesis 20, 1899-1903.

Sun, Y. (2000). Identification and characterization of genes responsive to apoptosis: Application of DNA chip technology and mRNA differential display. Histol Histopathol 15, 1271-1284.

Sun, Y. (2003). Targeting E3 ubiquitin ligases for cancer therapy. Cancer Biol Therapy 2, 623-629.

Sun, Y. (2006). E3 ubiquitin ligases as cancer targets and biomarkers. Neoplasia 8, 645-654.

Sun, Y. (2008). RNF7 (RING finger protein-7). Atlas Genet Cytogenet Oncol Haematol 12, 289-291.

Sun, Y., Bian, J., Wang, Y., and Jacobs, C. (1997). Activation of p53 transcriptional activity by 1,10 -phenanthroline, a metal chelator and redox sensitive compound. Oncogene 14, 385-393.

Sun, Y., Tan, M., Duan, H., and Swaroop, M. (2001). SAG/ROC/Rbx/Hrt, a zinc RING finger gene family: molecular cloning, biochemical properties, and biological functions. Antioxid Redox Signal 3, 635-650.

Sutterluty, H., Chatelain, E., Marti, A., Wirbelauer, C., Senften, M., Muller, U., and Krek, W. (1999). p45SKP2 promotes p27Kip1 degradation and induces $S$ phase in quiescent cells. Nature Cell Biol 1, 207-214.

Swaroop, M., Bian, J., Aviram, M., Duan, H., Bisgaier, C.L., Loo, J.A., and Sun, Y. (1999). Expression, purification, and biochemical characterization of SAG, a RING finger redox sensitive protein. Free Radicals Biol Med 27, 193-202.

Swaroop, M., Gosink, M., and Sun, Y. (2001). SAG/ROC2/Rbx2/Hrt2, a component of SCF E3 ubiquitin ligase: genomic structure, a splicing variant, and two family pseudogenes. DNA Cell Biol 20, 425-434.

Swaroop, M., Wang, Y., Miller, P., Duan, H., Jatkoe, T., Madore, S., and Sun, Y. (2000). Yeast homolog of human SAG/ROC2/R-
bx2/Hrt2 is essential for cell growth, but not for germination: Chip profiling implicates its role in cell cycle regulation. Oncogene 19, 2855-2866.

Swords, R.T., Kelly, K.R., Smith, P.G., Garnsey, J.J., Mahalingam, D., Medina, E., Oberheu, K., Padmanabhan, S., O'Dwyer, M., Nawrocki, S.T., et al. (2010). Inhibition of NEDD8-activating enzyme: a novel approach for the treatment of acute myeloid leukemia. Blood 115, 3796-3800.

Tan, M., Davis, S.W., Saunders, T.L., Zhu, Y., and Sun, Y. (2009). RBX1/ROC1 disruption results in early embryonic lethality due to proliferation failure, partially rescued by simultaneous loss of p27. Proc Natl Acad Sci U S A 106, 6203-6208.

Tan, M., Gallegos, J.R., Gu, Q., Huang, Y., Li, J., Jin, Y., Lu, H., and Sun, Y. (2006). SAG/ROC-SCFbeta-TrCP E3 ubiquitin ligase promotes pro-caspase-3 degradation as a mechanism of apoptosis protection. Neoplasia 8, 1042-1054.

Tan, M., Gu, Q., He, H., Pamarthy, D., Semenza, G.L., and Sun, Y. (2008). SAG/ROC2/RBX2 is a HIF-1 target gene that promotes HIF-1alpha ubiquitination and degradation. Oncogene 27, 1404-1411.

Tan, M., Li, Y., Yang, R., Xi, N., and Sun, Y. (2011a). Inactivation of SAG E3 ubiquitin ligase blocks embryonic stem cell differentiation and sensitizes leukemia cells to retinoid acid. PLoS One 6, e27726.

Tan, M., Zhao, Y., Kim, S.J., Liu, M., Jia, L., Saunders, T.L., Zhu, Y., and Sun, Y. (2011b). SAG/RBX2/ROC2 E3 Ubiquitin Ligase Is Essential for Vascular and Neural Development by Targeting NF1 for Degradation. Dev Cell 21, 1062-1076.

Tan, M., Zhu, Y., Kovacev, J., Zhao, Y., Pan, Z.Q., Spitz, D.R., and Sun, Y. (2010). Disruption of Sag/Rbx2/Roc2 induces radiosensitization by increasing ROS levels and blocking NF-kB activation in mouse embryonic stem cells. Free Radic Biol Med 49 976-983.

Tsvetkov, L.M., Yeh, K.-H., Lee, S.-J., Sun, H., and Zhang, H. (1999). p2 $7^{\text {kip1 }}$ ubiquitination and degradation is regulated by the $\mathrm{SCF}^{\text {skp2 }}$ complex through phosphorylated Thr187 in p27. Cur Biol 9, 661-664.

Vesterlund, M., Zadjali, F., Persson, T., Nielsen, M.L., Kessler, B.M., Norstedt, G., and Flores-Morales, A. (2011). The SOCS2 ubiquitin ligase complex regulates growth hormone receptor levels. PLoS One 6, e25358.

Viarengo, A., Burlando, B., Ceratto, N., and Panfoli, I. (2000). Antioxidant role of metallothioneins: a comparative overview. Cell Mol Biol 46, 407-417.

Wei, D., Li, H., Yu, J., Sebolt, J.T., Zhao, L., Lawrence, T.S., Smith, P.G., Morgan, M.A., and Sun, Y. (2012). Radiosensitization of human pancreatic cancer cells by MLN4924, an investigational NEDD8-activating enzyme inhibitor. Cancer Res 72, 282-293.

Wei, D., Morgan, M.A., Sun, Y. (2012). Radiosensitization of cancer cells by inactivation of cullin-RING E3 ubiquitin ligases. Transl Oncol 5, 305-312.

Wei, D., and Sun, Y. (2010). Small RING finger proteins RBX1 and RBX2 of SCF E3 ubiquitin ligases: the role in cancer and as cancer targets. Genes Cancer 1, 700-707.

Wei, W., Jin, J., Schlisio, S., Harper, J.W., and Kaelin, W.G., Jr. (2005). The v-Jun point mutation allows c-Jun to escape GSK3-dependent recognition and destruction by the Fbw7 
ubiquitin ligase. Cancer Cell 8, 25-33.

Welcker, M., and Clurman, B.E. (2008). FBW7 ubiquitin ligase: a tumour suppressor at the crossroads of cell division, growth and differentiation. Nat Rev Cancer 8, 83-93.

Willems, A.R., Schwab, M., and Tyers, M. (2004). A hitchhiker's guide to the cullin ubiquitin ligases: SCF and its kin. Biochim Biophys Acta 1695, 133-170.

Winston, J.T., Strack, P., Beer-Romero, P., Chu, C.Y., Elledge, S.J., and Harper, J.W. (1999). The SCFbeta-TRCP-ubiquitin ligase complex associates specifically with phosphorylated destruction motifs in IkappaBalpha and beta-catenin and stimulates IkappaBalpha ubiquitination in vitro. Genes Dev 13, 270-283.

Wrighton, K.H. (2011). Cell signalling: mTOR targets its own inhibitor. Nat Rev Mol Cell Biol 12, 769.

Wu, K., Fuchs, S.Y., Chen, A., Tan, P., Gomez, C., Ronai, Z., and Pan, Z.Q. (2000a). The SCF(HOS/beta-TRCP)-ROC1 E3 ubiquitin ligase utilizes two distinct domains within CUL1 for substrate targeting and ubiquitin ligation. Mol Cell Biol 20, 1382-1393.

Wu, K., Fuchs, S.Y., Chen, G., Tan, P., Gomez, C., Ronai, Z., and Pan, Z.-Q. (2000b). The SCF ${ }^{\text {HOS/b-TRCP }}-$ ROC1 E3 ubiquitin ligase utilizes two distinct domains within CUL1 for substrate targeting and ubiquitin ligation. Mol. Cell. Biol. 20, 1382-1393.

Yamaoka, S., Courtois, G., Bessia, C., Whiteside, S.T., Weil, R., Agou, F., Kirk, H.E., Kay, R.J., and Israel, A. (1998). Complementation cloning of NEMO, a component of the IkappaB kinase complex essential for NF-kappaB activation. Cell 93, 1231-1240.

Yang, D., Tan, M., Wang, G., and Sun, Y. (2012). The p21-dependent radiosensitization of human breast cancer cells by MLN4924, an investigational inhibitor of NEDD8 activating enzyme. PLoS One 7, e34079.

Yang, E.S., Huh, Y.J., and Park, J.W. (2010). Knockdown of sensitive to apoptosis gene by small interfering RNA enhances the sensitivity of PC3 cells toward actinomycin D and etoposide. Free Radic Res 44, 864-870.
Yang, E.S., and Park, J.W. (2006). Regulation of nitric oxide-induced apoptosis by sensitive to apoptosis gene protein. Free Radic Res 40, 279-284.

Yang, G.Y., Pang, L., Ge, H.L., Tan, M., Ye, W., Liu, X.H., Huang, F.P., Wu, D.C., Che, X.M., Song, Y., et al. (2001). Attenuation of ischemia-induced mouse brain injury by SAG, a redox- inducible antioxidant protein. J Cereb Blood Flow Metab 21, 722-733.

Yasukawa, T., Kamura, T., Kitajima, S., Conaway, R.C., Conaway, J.W., and Aso, T. (2008). Mammalian Elongin A complex mediates DNA-damage-induced ubiquitylation and degradation of Rpb1. EMBO J 27, 3256-3266.

Yoo, D.Y., Shin, B.N., Kim, I.H., Kim, D.W., Yoo, K.Y., Kim, W., Lee, C.H., Choi, J.H., Yoon, Y.S., Choi, S.Y., et al. (2012). Effects of sensitive to apoptosis gene protein on cell proliferation, neuroblast differentiation, and oxidative stress in the mouse dentate gyrus. Neurochem Res 37, 495-502.

Yu, X., Yu, Y., Liu, B., Luo, K., Kong, W., Mao, P., and Yu, X.F. (2003). Induction of APOBEC3G ubiquitination and degradation by an HIV-1 Vif-Cul5-SCF complex. Science 302, 1056-1060.

Zhao, L., Yue, P., Lonial, S., Khuri, F.R., and Sun, S.Y. (2011a). The NEDD8-activating enzyme inhibitor, MLN4924, cooperates with TRAIL to augment apoptosis through facilitating C-FLIP degradation in head and neck cancer cells. Mol Cancer Ther 10, 2415-2425.

Zhao, Y., and Sun, Y. (2012). Targeting the mTOR-DEPTOR Pathway by CRL E3 Ubiquitin Ligases: Therapeutic Application. Neoplasia 14, 360-367.

Zhao, Y., Xiong, X., Jia, L., and Sun, Y. (2012). Targeting Cullin-RING ligases by MLN4924 induces autophagy via modulating the HIF1-REDD1-TSC1-mTORC1-DEPTOR axis. Cell Death Dis 3, e386.

Zhao, Y., Xiong, X., and Sun, Y. (2011b). DEPTOR, an mTOR inhibitor, is a physiological substrate of SCF $\beta$ TrCP E3 ubiquitin ligase and regulates survival and autophagy. Mol Cell 44, 304-316. 